\title{
Note sugli anni ottanta: il caso italiano nel contesto internazionale
}

Notes sur les années quatre-vingt: le cas italien dans le contexte international Remarks on the eighties: the Italian case in the international context

Leonardo Casalino

\section{OpenEdition}

\section{Journals}

Edizione digitale

URL: http://journals.openedition.org/cei/303

DOI: $10.4000 /$ cei.303

ISSN: 2260-779X

\section{Editore}

UGA Éditions/Université Grenoble Alpes

Edizione cartacea

Data di pubblicazione: 15 mars 2012

Paginazione: 11-18

ISBN: 978-2-84310-222-6

ISSN: 1770-9571

\section{Notizia bibliografica digitale}




\title{
NOTE SUGLI ANNI OTTANTA: IL CASO ITALIANO NEL CONTESTO INTERNAZIONALE
}

\author{
Leonardo Casalino \\ Université Stendhal - Grenoble 3
}

Il I2 febbraio I980, l'Indipendent Commission for International Developmental Issues, più generalmente conosciuta come la North-South Commission, presieduta dall'ex-cancelliere tedesco Willy Brandt, presentò il proprio rapporto conclusivo al segretario generale delle Nazioni Unite a New York. Il titolo per esteso recitava Assicurare la sopravvivenza - Interesse comune dei paesi industrializzati e di quelli in via di sviluppo, ma il documento divenne generalmente noto come "rapporto Brandt" e costituisce, a giudizio di Marco Revelli «l'ultimo organico tentativo di dare una risposta "di sinistra" alla sfida emergente di un mondo sempre più globale» ${ }^{ }$.

Il rapporto indicava la necessità di combattere la fame e la povertà dei paesi poveri attraverso la formazione di un nuovo sistema economico mondiale capace d'integrare anche le nazioni del Terzo Mondo. Brandt introduceva il concetto di «responsabilità» globale: la quale avrebbe dovuto condurre a una ridistribuzione delle risorse e a una maggiore uguaglianza nel mondo. I paesi sviluppati dovevano comprendere, infatti, che una riduzione delle diseguaglianze a livello planetario avrebbe favorito non solo un ordine geopolitico più pacifico e un più equilibrato sviluppo umano, ma avrebbe anche offerto alle loro economie la possibilità di approfittare di una significativa crescita della domanda.

Un Occidente sempre più in difficoltà nel vendere i propri prodotti ne avrebbe potuto trarre notevoli vantaggi e, se la filosofia sociale del rapporto fosse stata seguita, la sinistra europea avrebbe potuto misurarsi con più forza con le grandi novità della globalizzazione nascente. Le cose, però,

I. M. Revelli, «La sinistra e la sfida neoliberista degli anni Ottanta», in Enciclopedia della sinistra europea nel XX secolo, a cura di A. Agosti, Roma, Editori Riuniti, 2000, p. 793. 
andarono diversamente e le indicazioni del rapporto furono largamente ignorate. Anzi, rapidamente, all'inizio degli anni Ottanta, sia negli Stati Uniti sia in Europa le strategie vincenti ebbero una fisionomia radicalmente opposta: riduzione dell'intervento pubblico in economia, ridimensionamento del ruolo e della forza dei sindacati, fiducia ideologica nella capacità del mercato di autoregolarsi.

In Inghilterra questa politica neo-liberista fu perseguita dal leader conservatore Margaret Thatcher, la quale nel 1979 sconfisse i laburisti accusandoli di essere appunto «il partito dell'intervento governativo nell'economia» e di essere «ostaggio» dei sindacati. A partire dal 198I il repubblicano Ronald Reagan, condurrà negli Stati Uniti una politica di larghe privatizzazioni, di detassazione per i più ricchi, di riduzione delle spese sociali, senza preoccuparsi delle inevitabili conseguenze sul piano dell'occupazione e della coesione sociale.

Per la destra dell'inizio degli anni Ottanta era fondamentale sfruttare la favorevole congiuntura politica per colpire il suo nemico principale: il blocco sociale costituito dal lavoro salariato, il quale andava sconfitto politicamente e frammentato socialmente attraverso i processi di ristrutturazione industriale spostando le fabbriche e le aziende in paesi dove era più basso il livello salariale e minori erano le garanzie sociali e sindacali. Si trattava di un modello radicalmente opposto a quello contenuto nel rapporto Brandt. Al keynesismo globale di quello, destinato a innescare processi di crescita delle singole economie nazionali, si contrapponeva una sorta di globalizzazione liberista destinata a gestire la crescita lenta delle società avanzate facendone pagare i prezzi ai paesi poveri e alle classi povere.

Le forze progressiste non compresero la vera natura di questi processi ritenendo che la vittoria della destra potesse ancora iscriversi a ragioni congiunturali e che si trattasse di una normale dinamica di alternanza di governo. Vi furono negli anni Ottanta delle lotte di resistenza in diversi paesi: la Fiat nell'autunno I980 in Italia, i siderurgici francesi della Lorena, i minatori inglesi. Tutte inesorabilmente sconfitte e che ristudiate oggi acquistano il significato di un'anticipazione a livello nazionale di quello che emerse con più chiarezza alla fine del decennio con la caduta del regime sovietico: la crisi di tutte le esperienze che avevano caratterizzato la storia delle sinistre novecentesche. Le quali non ressero l'urto della dissoluzione delle grandi concentrazioni operaie e dell'indebolimento dell'autonomia dello Stato-nazione provocato dalla globalizzazione.

Questi processi erano, in realtà, cominciati alla fine degli anni Sessanta, in un contesto internazionale caratterizzato da due superpotenze in competizione tra di loro, ma entrambe in grande difficoltà. Il primo che cercò di 
trovare una soluzione a questa situazione di disordine fu Henry Kissinger, il quale era convinto che la crisi non fosse legata al rialzo del prezzo del petrolio e che dunque bisognava evitare l'errore di ricorrere a politiche di protezionismo o di difesa delle singole monete. Bisognava invece agire sulla riduzione dei salari sulla limitazione dei conflitti sociali e sulla riduzione della spesa pubblica. Inoltre bisognava creare, a livello internazionale, degli organismi capaci di gestire un'economia sempre più mondializzata (FMI, Banca Mondiale) e sempre più lanciata verso lo sviluppo della finanza privata e di grandi imprese internazionali. All'inizio degli anni Ottanta, però, questo progetto dimostrava i suoi limiti: non era sufficiente ridurre i salari a vantaggio dei profitti, occorreva una ristrutturazione più ampia, un aumento della produttività e soprattutto l'individuazione di nuovi mercati. Per far questo occorreva creare un diverso ordine geopolitico, una nuova divisione internazionale del lavoro e integrare nello sviluppo una grande zona del mondo.

S'iniziò con l'America Latina e con golpes effettuati da militari formati in accademie militari USA e con consulenti economici che provenivano dalle università americane in cui si affermava il «pensiero unico liberista». In Asia si coinvolsero l'Indonesia (giustificando il massacro di mezzo milione di comunisti), Taiwan e la Corea, promovendo una riforma agraria per creare consenso e poi uno sviluppo industriale fondato sugli aiuti ricevuti per essere state basi militari durante la guerra in Vietnam. Il capolavoro politico di Kissinger fu però la politica verso la Cina comunista: dopo la rottura con l'URSS e il danno provocato dal ritiro dei tecnici sovietici, la rivoluzione culturale aveva trasformato Mosca nel nemico principale. Kissinger approfittò di queste tensioni avviando nel 1972 il riconoscimento della Cina da parte degli Stati Uniti. Un processo che porterà progressivamente alla nascita di alcune zone speciali dove poterono nascere imprese joint-venture, operanti sul mercato internazionale, le quali divennero degli elementi trainanti dell'economia del paese. Anche i contadini, organizzati in cooperative, ottennero il diritto di vendere i lori prodotti. Quando questa trasformazione si avviò Mao era ancora vivo, in seguito toccò a Deng proseguire nella riforma capitalistica. Nel frattempo l'URSS si chiudeva nella glaciazione immobilista dell'epoca brezneviana e cadeva nell'errore storico di accettare la sfida - impossibile per la tenuta della sua economia - del riarmo lanciata da Reagan: bomba al neutrone, scudo missilistico, guerre stellari. Il complesso militare industriale statunitense, finanziato da imprese private, accelerava il salto tecnologico e lanciava sul piano culturale il nuovo mito di una "missione americana", per riunificare il mondo in nome dell'estensione della democrazia. 
Un progetto che non trovò nel nuovo contesto internazionale nessuno capace di contrastarlo, soprattutto perché l'Europa non era ancora un soggetto politico e la sinistra era incapace di elaborare nuove idee. La Francia avrebbe potuto essere il solo paese capace di resistere, grazie all'eredità gollista e a uno Stato efficiente dotato degli strumenti adeguati per guidare l'economia. Nel 197I Mitterrand aveva avviato la rifondazione socialista, unificando sindacalisti e intellettuali senza partito e elaborando un programma comune con i comunisti. Il quale prevedeva: aumenti salariali, estensione della spesa pubblica, intervento pubblico per colmare il deficit, alcune nazionalizzazioni. Nulla invece era detto sulle questioni internazionali e in modo particolare sulla questione europea e questo fu un limite destinato a pesare al momento della prova di governo. La quale si svolse all'inizio degli anni Ottanta senza la necessaria mobilitazione dal basso, in grado di sostenerla quando i rapporti di forza internazionali - sottovalutati - fecero sentire il loro peso. Licenziamenti come risposta agli aumenti salariali, fuga di capitali, la speculazione contro il franco, difficoltà nel rapporto con i sindacati, rapidamente presero corpo le condizioni per giustificare una svolta radicale rispetto al programma iniziale, la quale era destinata a influenzare le scelte degli altri partiti socialisti europei - soprattutto nel Sud del continente - nel breve e nel lungo periodo. Una cosa, comunque, era chiara: in un mercato internazionale con sempre meno regole, una politica di stampo keynesiano in un solo paese, isolata e senza alleanze, era destinata alla sconfitta ${ }^{2}$.

Se il contesto internazionale era questo, le vicende italiane difficilmente avrebbero potuto prendere un'altra piega. Nel passaggio dagli anni Settanta agli anni Ottanta si stava chiudendo una «stagione della storia della Repubblica» dove "pur nella permanenza dei protagonisti, lo scenario è sostanzialmente nuovo, ed è al suo interno che si ripresentano questioni di fondo non risolte» ${ }^{3}$. Per De Felice la più importante delle novità era costituita dal venir meno della centralità della questione comunista, una sorta di anticipazione nostrana della situazione che si creerà in Europa nel I989 con la fine del bipolarismo. Un PCI, ormai legittimato, veniva nuovamente escluso dall'alleanze di governo per scelta politica e non a causa della sua identità. Il "preambolo" democristiano (cioè il rifiuto di continuare la politica di apertura verso i comunisti, che segnò il cambio di direzione

2. Per una ricostruzione minuziosa e articolata di questi processi mondiali a cavallo tra gli anni Settanta e Ottanta si veda L. Magri, Il sarto di Ulm. Una storia possibile del PCI, Milano, Il Saggiatore, 2009.

3. F. De Felice, La questione della nazione repubblicana, Roma-Bari, Laterza, 1999, p. 23 I. 
del partito e il passaggio all'opposizione interna della corrente di sinistra democristiana) e la svolta di Berlinguer allontanavano definitivamente i due più grandi partiti (la cui forza organizzativa non corrispondeva più a una capacità reale d'innovazione politica), liberando al centro del sistema politico uno spazio enorme di azione al partito socialista e ai partiti laici, nel loro caso, però, sproporzionato rispetto all'effettivo peso politico. La crisi della democrazia italiana ha qui una delle sue origini più evidenti.

Il PSI craxiano, il quale all'inizio era stato capace di mobilitare numerosi e prestigiosi intellettuali nello sforzo di elaborare un programma per "l'alternativa", diventò rapidamente il custode più geloso di questo nuovo principio di "esclusione" verso i comunisti, accusati di mancanza di credibilità, perché vide in esso lo strumento capace di provocare nel tempo un riequilibrio elettorale a suo favore. La "governabilità" diventò la nuova parola d'ordine, una governabilità che avrebbe dovuto essere assicurata da una grande riforma istituzionale, spesso evocata, ma mai seriamente delineata. Il risultato, messo bene in luce da Franco De Felice, fu quello di determinare una pericolosa separazione tra la teoria e la prassi, tra il riformismo decisionista annunciato e la pratica di una rigida alleanza con la destra democristiana. Qui invece, si possono ritrovare le origini più vicine di un fenomeno che si affermerà in seguito: la confusione tra politica e antipolitica di un ceto politico feroce custode dei propri privilegi e che non esita a cavalcare la protesta della cosiddetta "società civile" per difenderli e proteggerli anche nelle fasi di un'apparente radicale trasformazione.

L'ultimo Berlinguer fu il critico più spietato e lucido di questo nuovo sistema di potere, di una politica debole facilmente corruttibile e pericolosamente corruttrice; la sua critica, però, avrebbe avuto bisogno di essere accompagnata anche da una riforma profonda del suo partito, una riforma in grado di completare sul piano organizzativo la strategia politica delineata tra il I980 e il 1984: l'alternativa democratica, la questione morale, la lotta per la pace, l'autonomia da Mosca, la difesa delle radici classiste del PCI anche in una fase di difesa (solidarietà agli operai della Fiat nel I980, lotta contro il decreto della scala mobile). La sua morte nel giugno I984 interruppe bruscamente e tragicamente questo progetto e non possiamo sapere come si sarebbero svolte le vicende italiane se fosse sopravvissuto (come non possiamo dire come sarebbero andate le cose a livello internazionale se, sempre nel '84, anche il tentativo di riforma di Andropov non fosse stato bloccato dalla sua scomparsa). Ciò che sappiamo, invece, è che il nuovo gruppo dirigente che arrivò alla testa del partito - dopo la parentesi di Natta - si convinse, ben presto, che la sopravvivenza del PCI dipendesse dal suo accesso al governo del paese, convinzione che condizionò non 
poco la stessa gestione della svolta dell'Ottantanove con esiti, purtroppo, alquanto negativi.

Gli avvenimenti politici, però, non sono comprensibili senza riflettere sui mutamenti della società e del mondo del lavoro. Il passaggio dall'Italia industriale a quella postindustriale avvenne in un contesto nazionale in cui il prevalere di una diffusa industria manifatturiera determinò la nascita di un ceto medio soprattutto impiegatizio e commerciale. Il quale dipendeva largamente dall'assistenzialismo dei partiti. Mancava invece un forte settore finanziario, assicurativo, borsistico che avrebbe favorito la nascita di un diverso modello di modernizzazione. L'Italia continuava a dipendere dall'estero per il suo approvigionamento di energia e le situazioni di crisi di fabbriche furono risolte con il ricorso alla cassa integrazione destinata a pesare sui conti dello Stato.

Il paese accumulò, perciò, un grave ritardo nel campo dell'informatica, della chimica, delle telecomunicazioni e dell'industria farmaceutica. Prevalse un capitalismo familiare e il periodo di sviluppo economico tra il 1983 e il 1986 (con una crescita annuale costante del 2,9\% del Prodotto Interno Lordo) non fu utilizzato per correggere queste basi troppo fragili. Il debito pubblico crebbe a causa del sostegno pubblico alle aziende, dell'uso disinvolto della spesa in pratiche assistenzialiste e della diminuzione delle entrate fiscali (conseguenza della tolleranza verso l'evasione fiscale). L'ingresso nel Sistema Monetario Internazionale nel 1979 aveva limitato l'automia dei governi nell'utilizzare la svalutazione della lira come strumento di sostegno all'economia. Si scelse allora di attirare capitali dall'estero mantenendo tassi d'interesse più elevati rispetto agli altri paesi europei, permettendo agli speculatori d'investire a tassi d'interesse del IO-I5\%. Si creò così un sistema perverso, in cui lo Stato per la propria liquidità aveva bisogno di vendere i titoli di Stato, ma era poi obbligato a pagare interessi sempre piu' alti con gravi conseguenze sul debito pubblico.

L'acquisto di BOT assorbiva risorse che, in questo modo, non venivano utilizzate per investimenti produttivi, in una sorta di euforia generale che subì il suo primo trauma con la crisi della Borsa di New York del I9 ottobre 1987. I governi di pentapartito preferirono concentrarsi sulla lotta contro l'inflazione tollerando un meccanismo che creava una ricchezza artificiale. L'economia sommersa (lavoro nero, evasione fiscale) permise all'Italia di diventare la quinta potenza industriale del mondo, ma favorì, contemporaneamente, la formazione di gruppi sociali che grazie a queste distorsioni raggiunsero un livello di vita che non intendevano più rimettere in discussione. Era questa la ragione per la quale tutti i tentativi di riforma fiscale 
provocarono enormi resistenze e non furono fino in fondo appoggiati da una classe politica che fondava il proprio consenso sulla difesa di privilegi particolari. Alla fine degli anni '8o la formula politica del CAF (Craxi, Andreotti, Forlani), l'alleanza tra il Psi e la destra democristiana, tradusse la rinuncia definitiva dei partiti di governo a ogni progetto di modernizzazione del paese, consegnando al decennio successivo un intreccio pericoloso tra interessi corporativi, partiti e un contesto internazionale in profondo mutamento.

Negli anni Ottanta, in definitiva, avvenne una cesura decisiva nella storia italiana: fino a quel momento i processi d'integrazione e di sviluppo avevano convissuto con i vizi di fondo, irrisolti, del paese: il cattivo funzionamento dei servizi pubblici, l'espandersi dei poteri privati, una spesa pubblica fuori controllo, il cancro delle trame occulte e i loro legami con la criminalità organizzata. Vizi che emergevano in tutta la loro gravità nel momento in cui il sistema politico dimostrava di non essere più in grado di rinnovarsi. Alfio Mastropaolo ha riassunto questo processo con grande efficacia:

Il modello di democrazia proposto al cittadino medio, ma anche alle élites, era quello di una democrazia 'tutelare', la quale sdoppiandosi, sì li proteggeva con efficacia, ma al contempo li deresponsabilizzava. Di conseguenza quando si è trattato di elaborare nuovi principi di convivenza, al posto delle ormai antiquate appartenenze ideologiche e subculturali, la maggioranza degli italiani ha preferito subire conformisticamente il degrado della politica, sommando alle sue insostenibili inezie, alla sua sterile conflittualità, alla corruzione diffusa, all'assistenzialismo corporativo la propria tradizionale (e non inspiegabile) insofferenza nei confronti dello Stato e d'ogni regola certa e troppo stringente, per reinserirsi alfine in quei particolarismi, campanilismi e familismi, cui a suo modo, la democrazia bicefala era riuscito a sottrarlo ${ }^{4}$.

Si erano create le condizioni, cioè, per la nascita di una destra nuova capace di rappresentare l'ostilità di una gran parte della società italiana verso i valori e le regole delle democrazie di massa affermatisi in altri paesi occidentali. Valori e regole che vennero svuotati dal basso riducendo la politica a una semplice selezione dei governanti. Se altrove queste tendenze trovarono i loro rappresentanti in leaders come la Thatcher o Reagan, in Italia negli anni Ottanta - come ha osservato di nuovo Alfio Mastropaolo questa domanda di destra faticò a trovare uno sfogo. Ciò non impedì però una trasformazione sul piano culturale, il diffondersi - cioè - nella società di una richiesta di ordine che si accompagnava alla formazione di un blocco sociale unificato dalla critica contro lo stato sociale. Si trattava 
di un senso comune destinato a pesare non poco nel successo politico di Berlusconi nel '94. Successo che non sarebbe stato possibile senza i cambiamenti intervenuti nei primi anni ' 90 e che in questa sede non possiamo approfondire. Le tensioni degli anni Ottanta, i vizi irrisolti, il vincolo esterno della costruzione europea, avevano spinto il sistema al suo punto di rottura aprendo la strada all'intervento della magistratura. Alla crisi della politica si era pensato di sopperire aggrappandosi da un lato a principi quali la competenza, la serietà, la professionalità e, dall'altro, alla via referendaria per favorire processi diversi di selezione delle classi dirigenti. Ancora una volta, però,

l'errore politico è stato quello di non distinguere tra le intenzioni di chi mirava a bonificare la democrazia e riconvertire le istituzioni alle necessità di un paese profondamente cambiato e quelle di coloro il cui vero traguardo era stravolgere l'impianto istituzionale a vantaggio dello schieramento anti-welfare, predisponendo nuovi strumenti per meglio ghettizzare la sinistra e gli interessi da essa rappresentatis.

L'incapacità delle classi dirigenti liberali di far fronte allo sviluppo della democrazia di massa aveva prodotto negli anni Venti le conseguenze che conosciamo. Nel 1945 i partiti di massa avevano costruito un sistema, certo, imperfetto e fondato su profonde divisioni ideologiche, ma che aveva funzionato nei momenti di crisi trovando la sua forza nelle comuni origini antifasciste e che aveva permesso al paese uno sviluppo economico considerevole. Negli anni Ottanta, quando questo equilibrio era entrato definitivamente in crisi, il "caso italiano" fu di nuovo contraddistinto dal fallimento delle classi dirigenti di fronte alle novità che si delineavano. Il laboratorio italiano si preparava a fabbricare un nuovo modello politico e non certo dei migliori. Capire perché le cose siano andate così tra il I980 e il 1994 richiederà ancora molte ricerche e letture. Un esercizio utile anche per orientarsi meglio nelle tormentate vicende politiche di questi primi decenni del XXI secolo. 